\title{
Dynamic Economic/Environmental Dispatch Problem Considering Prohibited Operating Zones
}

\author{
Ahmed Torchani \\ College of Engineering, University of \\ Hail, Hail, Saudi Arabia and \\ University of Tunis, ENSIT, LISIER \\ Laboratory, Tunisia \\ tochahm@yahoo.fr
}

\author{
Attia Boudjemline \\ College of Engineering, \\ University of Hail, \\ Hail, Saudi Arabia \\ a_boudjemline@hotmail.com
}

\author{
Hatem Gasmi \\ College of Engineering, University of \\ Hail, Hail, Saudi Arabia and \\ University of Tunis El-Manar, \\ ENIT, Tunisia \\ gasmi_hatem@yahoo.fr
}

\begin{abstract}
Along with economic dispatch, emission dispatch has become a key problem under market conditions. Thus, the combination of the above problems in one problem called economic emission dispatch (EED) problem became inevitable. However, due to the dynamic nature of today's network loads, it is required to schedule the thermal unit outputs in real-time according to the variation of power demands during a certain time period. Within this context, this paper presents an elitist technique, the second version of the non-dominated sorting genetic algorithm (NSAGII) for solving the dynamic economic emission dispatch (DEED) problem. Several equality and inequality constraints, such as valve point loading effects, ramp rate limits and prohibited operating zones $(\mathrm{POZ})$, are taken into account. Therefore, the DEED problem is considered as a nonconvex optimization problem with multiple local minima with higher-order non-linearities and discontinuities. A fuzzy-based membership function value assignment method is suggested to provide the best compromise solution from the Pareto front. The effectiveness of the proposed approach is verified on the standard power system with ten thermal units.
\end{abstract}

Keywords-dynamic environmental/economic dispatch; prohibited operating zones; multi-objective optimization; nondominated sorting genetic algorithm

\section{INTRODUCTION}

In electric power systems, improvement of operation and planning has become more important under the current market conditions and several tools have been developed in this context $[1,2]$. Economic load dispatch (ED) is one of them. It aims to schedule the outputs of the committed generating units so as to minimize the total fuel cost under specific system equality and inequality constraints. This objective can no longer be considered alone due to severe environmental standards imposed by legislations. In this respect, the Clean Air Act Amendments have been applied in the USA to reduce pollution and atmospheric emissions such as sulfur oxides, $\mathrm{SO}_{\mathrm{X}}$, and nitrogen oxides, $\mathrm{NO}_{\mathrm{X}}$, caused by fossil-fueled thermal units [3, 4]. Hence, improvements in dispatching electric power must consider both monetary profits and reduced emissions of gaseous pollution. Thus, we are facing a bi-objective minimization problem, which has been frequently known as the static environmental/economic dispatch (SEED) problem. SEED can only handle a single loading condition at a particular time instant [3-8]. Due to the large variation of the load demand and dynamic nature of the power systems in recent years, it is mandatory to schedule the generator outputs in real time according to the variation of power demands over a certain time period. There are several formulations of this problem, known as the dynamic environmental/economic dispatch (DEED) problem [9-12]. Generally, DEED is a dynamic optimization problem having the same objectives as SEED over a time period subdivided into smaller time intervals with respect to the constraints imposed on system operation by generator ramp-rate limits. Time period and time intervals can be one day and one hour, respectively. Therefore, the operational decision at an hour may affect the operational decision at a later hour. Authors in [11-16] summarize several techniques for solving dispatch problems. Conventional methods, such as dynamic programming, nonlinear programming, network flow method, and interior point method [16] have been criticized as they are iterative, sensitive to initial solution and converge into local optimum solution. To overcome these difficulties, more recent works centered around artificial intelligence (AI), such as genetic algorithm [17], Tabu search [18], particle swarm optimization [19-20], simulated annealing [12], differential evolution [13] and bacterial 
foraging [14]. These techniques proved to have a clear edge over traditional methods in solving DEED problems without any or less restrictions on the shape of the objective functions curves where multiple Pareto-optimal solutions can be obtained in a single run. Most of the past studies have only focused on the SEED problem except for a few where the multi-objective DEED problem is considered [14]. In [14], prohibited operating zones, ramp rate limit constraints and valve point loading effects (VPLE) have been considered. Therefore, the DEED becomes highly nonlinear and with discontinuous and non-convex cost functions.

Within this context, this paper presents an elitist multiobjective approach for solving the DEED problem including POZ, valve point loading effects, and ramp rate limit constraints. This proposed method, called second version of the non-dominated sorting genetic algorithm (NSAGII), incorporates a crowding distance comparison at the end of each iteration in order to facilitate the convergence of the optimization algorithm to the real Pareto optimal front. In general terms, the contribution of this study is to show that the NSGA approach used frequently for solving continuous problems can be efficient for non-smooth and non-convex DEED problems if a non-domination sorting technique is incorporated in the optimization algorithm. In addition, the ramp rate limit constraints have been considered during transition from the last hour of a day to the first hour of the next. A fuzzy set theory [5] is used to extract the best compromise solution from the Pareto optimal front for the decision makers. The proposed approach was tested on a tenunit test system incorporating all above constraints. This approach showed a very competitive performance when compared with the original NSGA algorithm.

\section{PROBLEM FORMULATION}

The DEED problem is considered as a multi-objective problem (MOP). It aims to minimize simultaneously the total fuel cost and total emission of thermal units over a certain period of time subdivided into smaller time intervals. Several equality and inequality constraints are considered in the problem formulation. Considering a power system with $N$ generators, the total fuel cost function $C_{T}$ in $(\$ / \mathrm{h})$ including VPLE and emission in (ton/h) are, respectively described by (1) and (2) [20]:

$$
\begin{aligned}
& \left.C_{T}=\sum_{t=1}^{T} \sum_{i=1}^{N}\left[a_{i}+b_{i} P_{i}^{t}+c_{i}\left(P_{i}^{t}\right)^{2}+\mid d_{i} \sin \left\{e_{i}\left(P_{i}^{\min }-P_{i}^{t}\right)\right\}\right\}\right] \\
& E_{T}=\sum_{t=1}^{T} \sum_{i=1}^{N}\left[10^{-2}\left(\alpha_{i}+\beta_{i} P_{i}^{t}+\gamma_{i}\left(P_{i}^{t}\right)^{2}\right)+\eta_{i} \exp \left(\lambda_{i} P_{i}^{t}\right)\right]
\end{aligned}
$$

where, $P_{i}^{t}$ is real power output of the $i$-th unit at time $t . T$ is the number of hours. $a_{i}, b_{i}, c_{i}, d_{i}$ and $e_{i}$ are the cost coefficients of the $i$-th unit. $\alpha_{i}, \beta_{i}, \gamma_{i}, \eta_{i}$ and $\lambda_{i}$ are the emission coefficients of the $i$-th unit. Objective functions $C_{T}$ and $E_{T}$ are optimized subject to the constraints described below.

\section{A. Generation Limits}

$$
P_{i}^{\min } \leq P_{i}^{t} \leq P_{i}^{\max }, i=1, \ldots, N
$$

\section{B. Power Balance Constraints}

Total demand power $P_{D}^{t}$ and total losses $P_{L}^{t}$ must be covered at each interval of time $t$.

$$
\sum_{i=1}^{N} P_{i}^{t}-P_{D}^{t}-P_{L}^{t}=0, t=1, \ldots, T
$$

In this study, total losses are expressed as follows [20]:

$$
P_{L}^{t}=\sum_{i=1}^{N} \sum_{j=1}^{N} P_{i}^{t} B_{i j} P_{j}^{t}+\sum_{i=1}^{N} B_{o i} P_{i}^{t}+B_{o o}
$$

where $B_{i j}, B_{o i}, B_{o o}$ are called $B$ coefficients.

\section{Ramp Rate Limits}

$$
\begin{aligned}
& P_{i}^{t-1}-P_{i}^{t} \leq R_{i}^{\text {down }} \\
& P_{i}^{t}-P_{i}^{t-1} \leq R_{i}^{u p}
\end{aligned}
$$

where $R_{i}^{\text {down }}$ and $R_{i}^{u p}$ are the down and up rate limits of the $i$ th unit, respectively.

\section{Constraints Due to Prohibited Operating Zones}

$$
P_{i}^{t} \in\left\{\begin{array}{l}
P_{i}^{\min } \leq P_{i}^{t} \leq \underline{P}_{i}^{1} \\
\bar{P}_{i}^{k-1} \leq P_{i}^{t} \leq \underline{P}_{i}^{k}, k=2, \ldots, z_{i} \\
\bar{P}_{i}^{z_{i}} \leq P_{i}^{t} \leq P_{i}^{\max }
\end{array}\right.
$$

where $z_{i}$ is the number of prohibited operating zones for the $i$ th unit, and $\bar{P}_{i}^{k}$ and $\underline{P}_{i}^{k}$ are upper and lower bounds of the prohibited zone number $k$.

\section{IMPLEMENTATION OF THE PROPOSED METHOD}

Multi-objective evolutionary algorithms using nondominated sorting and sharing, such as NSGA and NPGA, have been criticized for the absence of elitism. Therefore, the second version of NSGA, called NSGAII [21] is utilized in this study for solving the DEED problem. In this approach, the sharing function approach is replaced with a crowded comparison. Initially, an offspring population $Q_{t}$ is created from the parent population $P_{t}$ at the $t$-th generation. Then, a combined population $R_{t}$ is formed:

$$
R_{t}=P_{t} \cup Q_{t}
$$

$R_{t}$ is sorted into different no-domination levels $F_{j}$. So, we can write:

$$
R_{t}=\bigcup_{j=1}^{r}\left(F_{j}\right)
$$


where, $r$ is the number of fronts. To offer a higher precision with reduced CPU time, this algorithm has been implemented using real-coded genetic algorithm in [5, 19].

\section{RESULTS AND SIMULATION}

The effectiveness of the proposed optimization algorithm for solving the DEED problem is assessed on the 10-unit system. All system data are taken from $[14,20]$. The B-loss coefficients are given below.

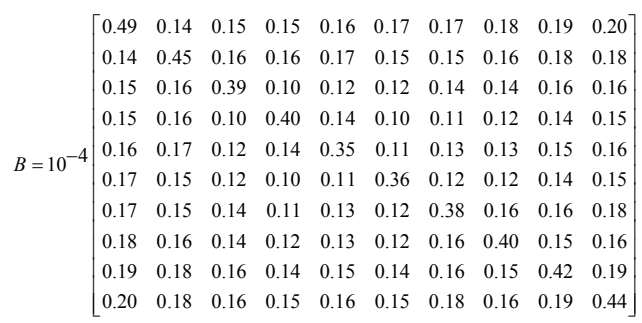

The NSGAII algorithm is implemented in MATLAB R2009a on a 64-bit operating system on a PC with an Intel i3$2370 \mathrm{M} \mathrm{CPU}$ at $2.40 \mathrm{GHz}$. The best compromise solution is generated from the Pareto front using a fuzzy based membership function value assignment method [5]. The NSGAII parameters to find the best Pareto set for the SEED problem have been chosen by trial and error and they were used for the DEED problem. In this study, the maximum number of generations and the population size were both chosen to be 200 .

- Test case 1: The SEED problem for the ten-unit system with $P_{D}=1036 \mathrm{MW}$ was considered in this case. Optimal outputs of thermal units for best cost, best emission and best compromise solution have been computed using the proposed optimization algorithm. Results have been compared with those obtained using NSGA.

- Test case 2: The DEED for the test system over a 24-hour time horizon was solved under all previous constraints. POZ limits in MW shown in Table I are taken from [22]. Therefore the problem will be more complicated with discontinuities. The hourly variation of the load is depicted in Figure 1.

TABLE I. UNIT OPERATING LIMITS IN MW

\begin{tabular}{|c|c|c|c|c|c|}
\hline Unit & $\boldsymbol{P}_{\boldsymbol{i}}^{\min }$ & $\boldsymbol{P}_{\boldsymbol{i}}^{\mathbf{m a x}}$ & $\boldsymbol{R}_{\boldsymbol{i}}^{\text {down }}$ & $\boldsymbol{R}_{\boldsymbol{i}}^{\text {up }}$ & Prohibited zone \\
\hline 1 & 150 & 470 & 80 & 80 & {$[150165],[448453]$} \\
\hline 2 & 135 & 470 & 80 & 80 & {$[90110],[240250]$} \\
\hline 3 & 73 & 340 & 80 & 80 & - \\
\hline 4 & 60 & 300 & 50 & 50 & - \\
\hline 5 & 73 & 243 & 50 & 50 & - \\
\hline 6 & 57 & 160 & 50 & 50 & - \\
\hline 7 & 20 & 130 & 30 & 30 & - \\
\hline 8 & 47 & 120 & 30 & 30 & {$[2030],[4045]$} \\
\hline 9 & 20 & 80 & 30 & 30 & - \\
\hline 10 & 10 & 55 & 30 & 30 & {$[1217],[3545]$} \\
\hline
\end{tabular}

\section{A. SEED Problem: Test Case 1}

For the validation of the proposed algorithm, a comparison with the first version of NSGA is suggested in this case. From
Figure 1, it is clear that NSGAII provides the best results and it has better diversity characteristics of non-dominated solutions. From Table II, the minimum fuel cost and emission provided by NSGAII are $\$ 61,775.44$ and $3,785.471 \mathrm{~b}$ respectively. Moreover, the highest value of the fuel cost is found for minimum emission and the highest value of the emission corresponds to the minimum fuel cost since they are conflicting objective functions.

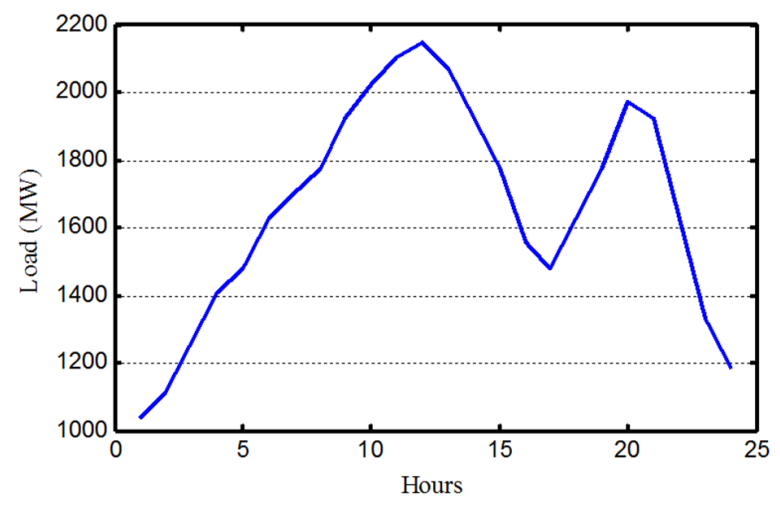

Fig. 1. Hourly load variation

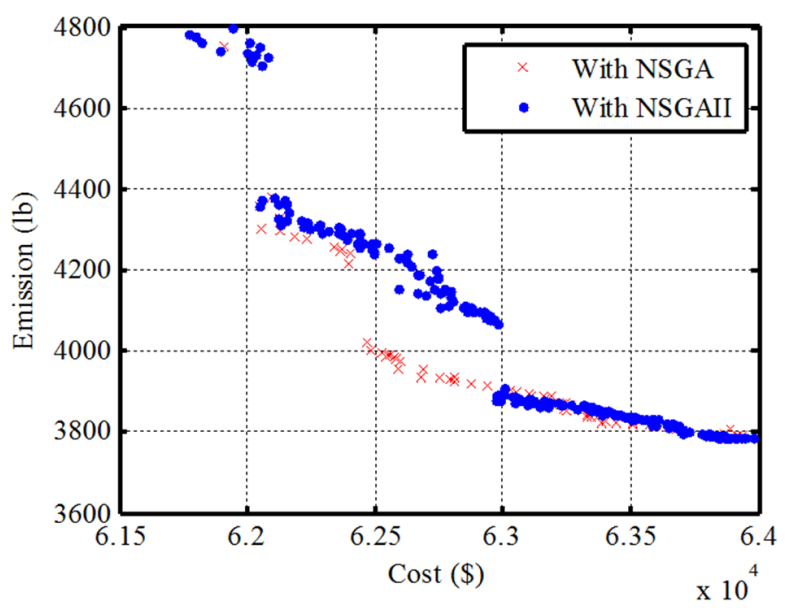

Fig. 2. Pareto solutions with NSGAII and NSGA (case 1)

\section{B. DEED Problem Considering All Constraints: Test Case 2}

In this case, the generation output of unit at each hour has been adjusted considering POZ. Consequently, discontinuities are introduced in cost and emission curves corresponding to the POZ. The hourly evolution of the optimum generations using the proposed algorithm for minimum cost is shown in Figure 3. It is clear that the outputs of all units are maximum at hour 12 which corresponds to the maximum load (2150MW). In this sub-section, optimum solution for minimum emission is not displayed due to the space limitation. Table III shows the compromise solution extracted from the Pareto solutions. It is clear that the proposed scheduling of generations satisfies all previous constraints. 


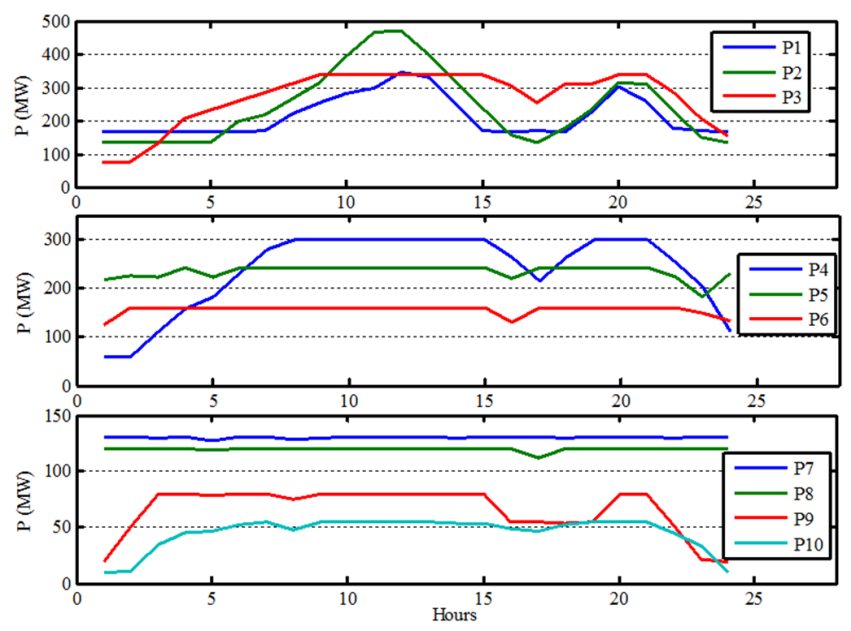

Fig. 3. Hourly evolution of the optimum solution for minimum cost

\section{CONCLUSION}

The DEED problem is one of the most crucial issues to be solved in the power system field. It has a great importance in reducing emission of harmful gases and saving energy. In this study, the DEED problem has been formulated as a biobjective optimization problem with nonlinear constraints including VPLE, ramp rate limits and prohibited operating zones. The second version of the non-dominated sorting genetic algorithm (NSGAII) has been suggested for solving the DEED problem for 24-hour dispatch intervals. To demonstrate the effectiveness of the proposed approach, the standard power system with ten thermal units was used. Various cases with different levels of complexity and discontinuity have been considered. The results of the proposed approach are significantly improved when compared with NSGA. In addition, this approach has the capacity to optimize any number of objective functions simultaneously and generate the Pareto front in a single run. Therefore, other objectives can be included in the main problem such as voltage drop and real power losses.

TABLE II. OPTIMUM SOLUTIONS FOR CASE 1

\begin{tabular}{|c|c|c|c|c|c|c|}
\hline & \multicolumn{2}{|c|}{$\begin{array}{c}\text { Minimum } \\
\text { fuel cost }\end{array}$} & \multicolumn{2}{c|}{$\begin{array}{c}\text { Minimum } \\
\text { emission }\end{array}$} & \multicolumn{2}{c|}{$\begin{array}{c}\text { Best compromise } \\
\text { solution }\end{array}$} \\
\hline Method & NSAGII & NSGA & NSGAII & NSGA & NSGAII & NSGA \\
\hline P1 & 165.657 & 165.304 & 165.465 & 165.277 & 165.092 & 165.400 \\
\hline P2 & 135.000 & 135.000 & 136.471 & 138.754 & 135.000 & 135.073 \\
\hline P3 & 73.0000 & 73.0000 & 85.943 & 89.8068 & 78.3817 & 73.0000 \\
\hline P4 & 60.0000 & 60.0000 & 87.8645 & 88.9776 & 85.9374 & 77.8433 \\
\hline P5 & 221.551 & 224.103 & 135.325 & 124.263 & 123.237 & 131.320 \\
\hline P6 & 120.835 & 118.647 & 126.733 & 126.764 & 128.576 & 124.444 \\
\hline P7 & 130.000 & 130.000 & 91.4739 & 97.8403 & 129.019 & 130.000 \\
\hline P8 & 120.000 & 120.000 & 91.9147 & 89.3054 & 84.4827 & 120.000 \\
\hline P9 & 20.0000 & 20.0000 & 79.6906 & 79.8941 & 79.5035 & 51.7425 \\
\hline P10 & 10.0000 & 10.0000 & 55 & 55.0000 & 46.6442 & 47.0783 \\
\hline Cost (\$/h) & 61775.4 & 61802.6 & 63914.4 & 63905.5 & 62974.5 & 62486.2 \\
\hline $\begin{array}{c}\text { Emission } \\
\text { (lb/h) }\end{array}$ & 4781.79 & 4800.55 & 3785.47 & 3785.51 & 3880.30 & 3998.43 \\
\hline
\end{tabular}

\section{ACKNOWLEDGMENT}

The present work was undertaken within the research project (No: 160803), funded by the Deanship of Scientific Research, at the University of Hail, which is gratefully acknowledged.

TABLE III. BEST COMPROMISE SOLUTION OF DEED FOR TEST CASE 2

\begin{tabular}{|c|c|c|c|c|c|c|c|c|c|c|}
\hline Hour & P1 & P2 & P3 & P4 & P5 & P6 & P7 & P8 & P9 & P10 \\
\hline 1 & 165.0185 & 136.1190 & 73.0000 & 88.1843 & 125.1063 & 121.1142 & 130.0000 & 119.4657 & 52.5923 & 45.2700 \\
\hline 2 & 165.1026 & 135.1579 & 81.5542 & 108.405 & 173.2150 & 122.5238 & 100.0000 & 120.0000 & 80.0000 & 46.7179 \\
\hline 3 & 165.0594 & 135.7696 & 130.6567 & 127.3521 & 183.7788 & 159.8147 & 129.6961 & 120.0000 & 80.0000 & 54.5710 \\
\hline 4 & 165.5635 & 182.8389 & 164.0515 & 160.2915 & 225.3338 & 159.3824 & 130.0000 & 119.8330 & 79.8469 & 54.9029 \\
\hline 5 & 165.4107 & 202.1567 & 185.8223 & 182.477 & 240.2090 & 159.8194 & 129.8451 & 119.7818 & 79.8544 & 54.6686 \\
\hline 6 & 205.1453 & 218.9835 & 245.006 & 228.7587 & 243.0000 & 160.0000 & 121.126 & 120.0000 & 80.0000 & 55.0000 \\
\hline 7 & 200.0936 & 220.9863 & 294.5041 & 278.7587 & 243.0000 & 160.0000 & 106.1606 & 120.0000 & 77.1945 & 55.0000 \\
\hline 8 & 232.4056 & 297.4300 & 259.7886 & 263.0712 & 243.0000 & 157.1222 & 127.8133 & 120.0000 & 80.0000 & 55.0000 \\
\hline 9 & 295.7609 & 309.5763 & 339.7886 & 263.2257 & 241.7996 & 160.0000 & 130.0000 & 120.0000 & 80.0000 & 55.0000 \\
\hline 10 & 317.6374 & 355.8988 & 340.0000 & 300.0000 & 243.0000 & 160.0000 & 130.0000 & 120.0000 & 80.0000 & 55.0000 \\
\hline 11 & 358.3159 & 412.7551 & 340.0000 & 300.0000 & 243.0000 & 160.0000 & 130.0000 & 120.0000 & 74.8769 & 55.0000 \\
\hline 12 & 379.7618 & 434.7244 & 339.9887 & 299.9990 & 242.9959 & 160.0000 & 129.9935 & 119.9976 & 80.0000 & 54.9757 \\
\hline 13 & 346.8023 & 381.7131 & 340.0000 & 299.9520 & 242.9962 & 159.9909 & 130.0000 & 119.977 & 79.9899 & 55.0000 \\
\hline 14 & 302.4077 & 308.1888 & 300.5633 & 295.9688 & 243.0000 & 160.0000 & 130.0000 & 119.9821 & 80.0000 & 55.0000 \\
\hline 15 & 228.2163 & 264.2944 & 285.2806 & 270.8877 & 243.0000 & 159.4424 & 129.6214 & 119.887 & 79.9751 & 54.5439 \\
\hline 16 & 165.3236 & 222.6713 & 209.3282 & 239.4035 & 243.0000 & 159.4886 & 129.903 & 120.0000 & 54.6538 & 54.5022 \\
\hline 17 & 165.1747 & 216.9418 & 186.1643 & 189.4035 & 242.8615 & 159.9508 & 129.8344 & 119.8230 & 55.0000 & 55.0000 \\
\hline 18 & 226.6097 & 223.5607 & 229.9497 & 234.5908 & 242.8186 & 160.0000 & 130.0000 & 119.9758 & 55.0000 & 54.7545 \\
\hline 19 & 239.3959 & 299.4056 & 276.9271 & 258.0446 & 242.9016 & 159.8063 & 129.7669 & 119.8321 & 54.7896 & 54.8522 \\
\hline 20 & 276.0364 & 342.8300 & 340.0000 & 300.0000 & 243.0000 & 160.0000 & 130.0000 & 120.0000 & 80.0000 & 55.0000 \\
\hline 21 & 302.8172 & 308.8301 & 298.5865 & 298.2182 & 242.3453 & 159.9713 & 129.7167 & 119.7893 & 80.0000 & 54.8529 \\
\hline 22 & 224.9485 & 228.8301 & 218.5865 & 258.7530 & 209.6452 & 160.0000 & 130.0000 & 120.0000 & 80.0000 & 46.5327 \\
\hline 23 & 165.4124 & 149.0684 & 141.616 & 209.7076 & 166.2512 & 157.8307 & 128.9253 & 119.6998 & 79.8154 & 45.7563 \\
\hline 24 & 165.5672 & 135.0000 & 73.0000 & 138.1843 & 175.1063 & 145.5599 & 123.5542 & 120.0000 & 80.0000 & 53.6633 \\
\hline Total cost $(\$)$ & \multicolumn{10}{|c|}{2526555.7207} \\
\hline Total emission (lb) & \multicolumn{10}{|c|}{302900.8703} \\
\hline Total losses (MW) & \multicolumn{10}{|c|}{1301.8534} \\
\hline
\end{tabular}




\section{REFERENCES}

[1] F. Milano, "An open source power system analysis toolbox", IEEE Transactions on Power Systems, Vol. 20, No. 3, pp. 1199-1206, 2005

[2] R. D. Zimmerman, C. E. M. Sanchez, R. J. Thomas, "Matpower steadystate operations, planning and analysis tools for power systems research and education", IEEE Transactions on Power Systems, Vol. 26, No. 1, pp. 12-19, 2011

[3] S. Boudab, N. Golea, "Combined economic-emission dispatch problem: Dynamic neural networks solution approach", Journal of Renewable and Sustainable Energy, Vol. 9, No. 3, Article ID 035503, 2017

[4] M. Basu, "Economic environmental dispatch using multi-objective differential evolution", Applied Soft Computing, Vol. 11, No. 2, pp. 2845-2853, 2011

[5] M. A. Abido, "Multiobjective evolutionary algorithms for electric power dispatch problem", IEEE Transactions on Evolutionary Computation, Vol. 10, No. 3, pp. 315-329, 2006

[6] S. Sivasubramani, K. S. Swarup, "Environmental/economic dispatch using multi-objective harmony search algorithm", Electric Power Systems Research, Vol. 81, No. 9, pp. 1778-1785, 2011

[7] G. C. Liao, "Solve environmental economic dispatch of smart microgrid containing distributed generation system-using chaotic quantum genetic algorithm", International Journal of Electrical Power \& Energy Systems, Vol. 43, No. 1, pp. 779-787, 2012

[8] B. Hadji, B. Mahdad, K. Srairi, N. Mancer, "Multi-objective economic emission dispatch solution using dance bee colony with dynamic step size”, Energy Procedia, Vol. 74, pp. 65-76, 2015

[9] K. Tlijani, T. Guesmi, H. H. Abdallah, "Extended dynamic economic environmental dispatch using multi-objective particle swarm optimization", International Journal on Electrical Engineering and Informatics, Vol. 8, No. 1, pp. 117-131, 2016

[10] H. Ma, Z. Yang, P. You, M. Fei, "Multi-objective biogeography-based optimization for dynamic economic emission load dispatch considering plug-in electric vehicles charging", Energy, Vol. 135, pp. 101-111, 2017

[11] S. Hemamalini, S. P. Simon, "Dynamic economic dispatch using artificial bee colony algorithm for units with valve-point effect", European Transactions on Electrical Power, Vol. 21, No. 1, pp. 70-81, 2011

[12] C. K. Panigrahi, P. K. Chattopadhyay, R. N. Chakrabarti, M. Basu, "Simulated annealing technique for dynamic economic dispatch", Electric Power Components and Systems, Vol. 34, No. 5, pp. 577-586, 2006

[13] R. Balamurugan, S. Subramanian, "An improved differential evolution based dynamic economic dispatch with nonsmooth fuel cost function", Journal of Electrical Systems, Vol. 3, No. 3, pp. 151-161, 2007

[14] N. Pandit, A. Tripathi, S. Tapaswi, M. Pandit, "An improved bacterial foraging algorithm for combined static/dynamic environmental economic dispatch", Applied Soft Computing, Vol. 12, No. 11, pp. 3500-3513, 2012

[15] H. Rezaie, M. H. K. Rahbar, B. Vahidi, H. Rastegar, "Solution of combined economic and emission dispatch problem using anovel chaotic improved harmony search algorithm", Journal of Computational Design and Engineering, Vol. 6, No. 3, pp. 447-467, 2019

[16] G. Irisarri, L. M. Kimball, K. A. Clements, A. Bagchi, P. W. Davis, "Economic dispatch with network and ramping constraints via interior point methods", IEEE Transactions on Power Systems, Vol. 13, No. 1, pp. 236-242, 1998

[17] S. Ganjefar, M. Tofighi, "Dynamic economic dispatch solution using an improved genetic algorithm with non-stationary penalty functions", European Transactions on Electrical Power, Vol. 21, No. 3, pp. 1480 1492, 2011

[18] W. M. Lin, F. S. Cheng, M. T. Tsay, "An improved tabu search for economic dispatch with multiple minima”, IEEE Transactions on Power Systems, Vol. 17, No. 1, pp. 108-112, 2002

[19] K. Mason, J. Duggan, E. Howley, "Multi-objective dynamic economic emission dispatch using particle swarm optimisation variants", Neurocomputing, Vol. 270, pp. 188-197, 2017
[20] M. Basu, "Particle swarm optimization based goal-attainment method for dynamic economic emission dispatch", Electric Power Components and Systems, Vol. 34, No. 9, pp. 1015-1025, 2006

[21] K. Deb, A. Pratap, S. Agarwal, T. Meyarivan, "A fast and elitist multiobjective genetic algorithm: NSGA-II", IEEE Transactions on Evolutionary Computation, Vol. 6, No. 2, pp. 182-197, 2002

[22] M. Z. Jahromi, M. M. H. Bioki, M. Rashidinejad, R. Fadaeinedjad, "Solution to the unit commitment problem using an artificial neural network", Turkish Journal of Electrical Engineering and Computer Sciences, Vol. 21, pp. 198-212, 2013 